\title{
Solution Rectification of Slider-Crank Mechanisms with Transmission Angle Control
}

\author{
Wilhelm, Shawn R. \\ University of Minnesota \\ 111 Church St SE \\ Minneapolis, MN 55455 \\ Wilh0141@umn.edu \\ Sullivan, Thomas \\ University of Minnesota \\ 111 Church St SE \\ Minneapolis, MN 55455 \\ sullivth@gmail.com \\ Van de Ven, James D. \\ University of Minnesota \\ 111 Church St SE \\ Minneapolis, MN 55455 \\ vandeven@umn.edu
}

\begin{abstract}
Transmission angle control is well studied for both crank rocker and Grashof crank slider mechanisms. However, non-Grashof slider-crank mechanisms have received less attention. For these mechanisms, the fully rotatable crank constraint is removed increasing the complexity of the problem. Furthermore, methods of linkage synthesis by optimization are becoming more prevalent. When optimizing a mechanism, it is important to prevent the evaluation of non-constructible and undesirable linkages. Presented here is a method of solution rectification by means of transmission angle control for slidercrank mechanisms which can be used to parameterize a problem to prevent the evaluation of invalid linkages. The solution is kept general to include fully rotatable crank slider mechanisms as well. The solution considers both crank driven and slider driven mechanisms as well as a reversible driver mechanism. An example is provided to demonstrate the method. It will be shown that by defining a transmission angle, valid linkages can be generated preventing the evaluation of invalid solutions.
\end{abstract}

\section{INTRODUCTION}

In mechanism synthesis, defects occur that often result in unusable solutions and wasted computational effort. Solution rectification is a method of detecting and preventing such defects during synthesis and has been studied for numerous linkage topologies. This approach eliminates areas of the solution space that would result in linkages containing branch defects, circuit defects, order defects, or low transmission 
angles. Bali and Chand provide a comprehensive literature review of solution rectification, including transmission angle control in mechanisms [1,2]. In this paper, the complete range of motion of planar slider-crank mechanisms is described, as well as a method of solution rectification that includes transmission angle control.

Traditionally, the transmission angle is used to evaluate the quality of force and torque transmission through a fourbar linkage. Additionally, a number of authors have developed transmission quality indices to predict linkage performance such as the jointforce index analysis defined by Holte and Chase, the force transmission index, and more recently the effective force ratio [3-5]. In this paper, the context of linkage optimization is considered where the energy loss due to joint friction is an optimization objective as seen in [6-8]. This method proves useful for high-speed mechanisms, where the transmission angle is not a direct indicator of performance.

One of the challenges of optimization problems is maintaining generality without over-constraining the problem. It is possible to start an optimization which becomes trapped in an area of invalid solutions. Without valid solutions with which to construct a gradient, or create a functioning population in a GA method, the optimization will fail. It is useful to reduce the solution space and time to an optimal solution by eliminating computation time for invalid solutions and, for an evolutionary optimization, increasing the number of valid solutions in the population [9-14]. By defining the feasible range of motion of slider-crank mechanisms, the solution space is reduced to only include constructible mechanisms. This solution space can be reduced further by applying transmission angle control.

While the transmission angle rectification of four-bar mechanisms is well studied $[15,16]$, non-Grashof slider-crank mechanisms have received less attention. Previous works have shown transmission angle control of offset, Grashof, crank-slider mechanisms [17,18]. However, a solution for an offset, non-Grashof, slider-crank mechanisms has not yet been presented. Without the Grashof constraint, in which the input link completes a full revolution, the problem becomes more complex as more variables are introduced.

In this paper, a method is presented to determine the valid positions of slider-crank mechanisms, given a set of link lengths, a minimum transmission angle, and assuming a horizontal slider axis. The solution applies to both Grashof and non-Grashof slider-crank mechanisms. In section two, the method of defining the range of motion of a slide-crank mechanism with transmission angle control is presented. In section three, an example is given to demonstrate the method and provide clarification to the reader. Finally, concluding remarks are provided about the applicability of the proposed methods.

In this section, the linkage geometry and problem definition are described, and then the solution rectification method is described. Since the transmission angle depends on the driven link, the method is developed for crank driven, slider driven linkages, and their combination is used for switch mode linkages. 


\subsection{Linkage Geometry Definition}

With reference to Figure 1, the linkage geometry is defined by the length of the crank, $r$, the coupler link length, $l$, and the slider offset distance, $H$. The slider is assumed to travel parallel to the $x$-axis. The position of the mechanism is defined by the piston position, $X$, and the angle of the crank, $\theta$. Here the mechanism is parameterized by $\theta$, but could equally be defined by $X$.

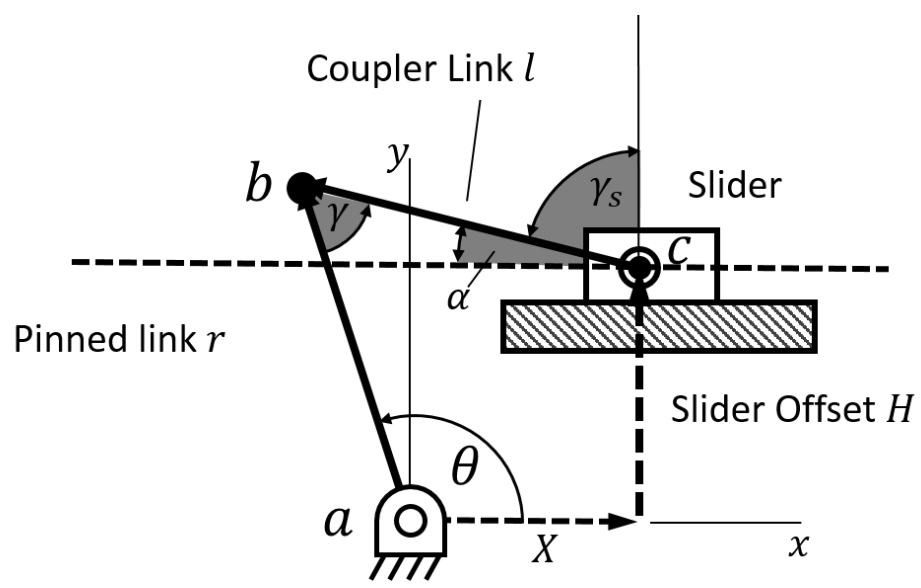

FIGURE 1 LINKAGE GEOMETRY

In this system, there are two transmission angle constraints to be specified depending on how the linkage is driven. If the slider is the input to the mechanism, the minimum transmission angle between the crank and coupler link, $\gamma$, is to be specified. If the crank is the input, the minimum transmission angle between the slider and coupler link, $\gamma_{s}$, is specified. If there is a reversal of driver and driven link, both angles are specified in order to control the effectiveness of the mechanism. To provide a more intuitive representation of the transmission quality at the slider joint, the pressure angle, $\alpha$, which is equal to $\left|\frac{\pi}{2}-\gamma_{s}\right|$ is used in the derivation.

The formulation presented in this paper assumes that $\alpha$ and $\gamma$ are acute and positive for consistency with the definition of transmission angle. A minimum transmission angle of zero is also allowed as well which can be used to define the unconstrained range of motion of the slider-crank. Since the transmission angle $\gamma$ is the acute angle between the crank and coupler link, there are two equivalent angles which are used in the derivation where $\gamma_{\min }=\gamma$ and $\gamma_{\max }=\pi-\gamma$. There is no such equivalency for the pressure angle, $\alpha$. It is assumed and that the slider is on the right side of the crank link $r$ and $H \geq 0$. Any other situation is realizable by reflection over the $y$ and/or $x$ axes.

Bounds on $\theta$ are determined with regards to the $\gamma$ and $\alpha$ constraints independently. If both constraints are to be considered simultaneously, these bounds are then compared to determine which values are limiting. 


\subsection{Slider Driven Mechanism}

The transmission angle is measured with reference to the output link of a mechanism. In the case of a slider driven mechanism the angle $\gamma$ is constrained. This section describes the solution rectification of a slider driven mechanism. From the defined linkage geometry and transmission angle requirements, a solution space of valid slider positions is created. When considering the maximum and minimum values of $\gamma$, an annulus, seen in Figure 2 , can be created with interior radius $k_{\min }$ and exterior radius $k_{\text {max }}$, defined by:

$$
k=\sqrt{r^{2}+l^{2}-2 r l \cos (\gamma)}
$$

where $k_{\min }$ and $k_{\max }$ are found using $\gamma_{\min }$ and $\gamma_{\max }$ respectively.

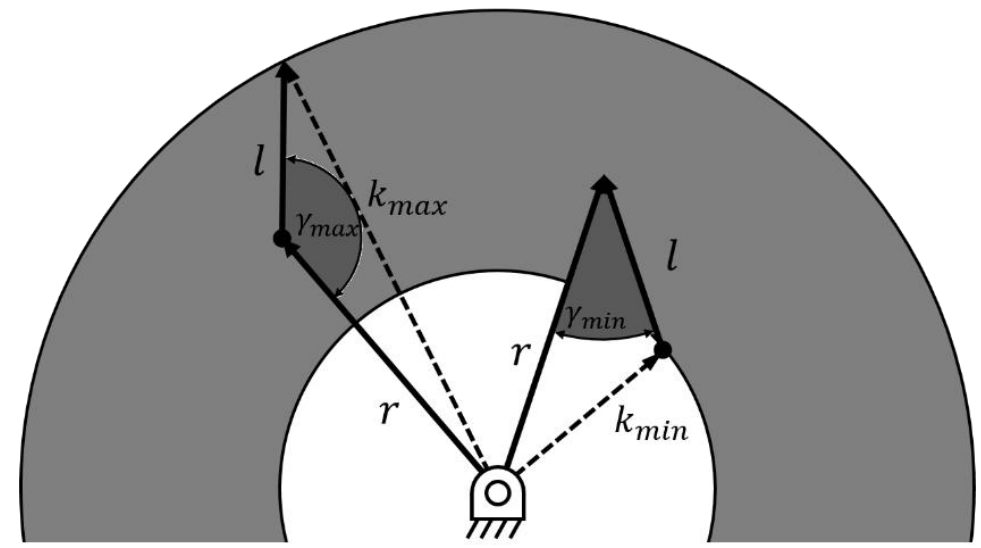

\section{FIGURE 2 ANNULUS DEFINING SOLUTION SPACE DEFINED BY TRANSMISSION ANGLE $\gamma$}

The slider offset distance, $H$ must be defined such that the resulting axis of slide passes through this annulus. There are three possible outcomes for a given value of $H$ :

Case A: If $H \geq k_{\max }$, the intersection is empty or at a single point, resulting in no useful linkages.

Case $\mathrm{B}$ : If $k_{\min } \leq H<k_{\max }$, the intersection is a continuous segment.

Case C: If $H<k_{\text {min }}$, the intersection is a pair of line segments.

Case C, depicted in Figure 3, is the most general case and is used to define the solution space for slider driven mechanisms. The two segments are mirrored about the $y$ axis resulting in a right and left segment solution space. This nomenclature is used throughout to differentiate these two spaces. Using the $k$ values found previously, the points of intersection between the segments and annulus can be found. These slider segment lengths are defined by the right triangle $\Delta k H X$ yielding the equation:

$$
X= \pm \sqrt{k^{2}-H^{2}}
$$

where $k$ is either $k_{\min }$ or $k_{\max } . X$ defines the right side segment while $-X$ defines the left. 


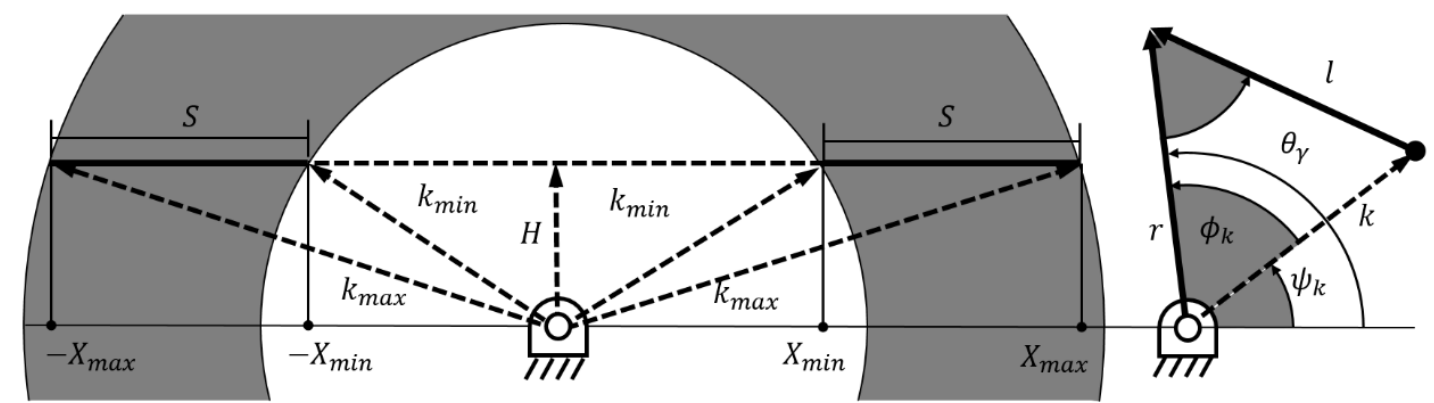

FIGURE 3 DEFINING VALID SLIDER POSITIONS CONSIDERING $\gamma$ CONSTRAINTS

The slider segment length is defined as:

$$
S=X_{\max }-X_{\min }
$$

There are typically four configurations possible of a slider-crank mechanism for the same link lengths. The slider can either be to the left or the right of a vertical line drawn through coupler pivot $b$, however these mechanisms are simply mirrored about the $y$ axis. The crank can either be above or below a line drawn between the crank pivot $a$ and the slider pin $c$. Assuming the slider is to the right side of the crank link, the above and below configurations are defined by right and left segments respectively. The left segment is in fact a mirror of the below configuration of the right segment. The slidercrank mechanism is presented this way for consistency with the other cases, and for a more general solution.

For each of the bounding points, there is an associated angle between the $x$-axis and the $k$ line defined by:

$$
\psi_{k}=\operatorname{atan} 2(H, X)
$$

where a unique value is found for each of the four $X$ points and atan 2 is the four quadrant inverse tangent function.

The angle between each $k$ line and the crank is described by:

$$
\phi_{k}=\operatorname{acos}\left(\frac{r^{2}+k^{2}-l^{2}}{2 r k}\right)
$$

The bounding angles of the crank are expressed as:

$$
\theta_{\gamma}=\psi_{k} \pm \phi_{k}
$$

where $\phi_{k}$ is added to $\psi_{k}$ for the right segment and subtracted for the left segment. These calculations result in a total of 4 bounding angles defining the right and left segment solution spaces. These four bounding values of $\theta_{\gamma}$ are depicted in Figure 4 for an arbitrary linkage. 


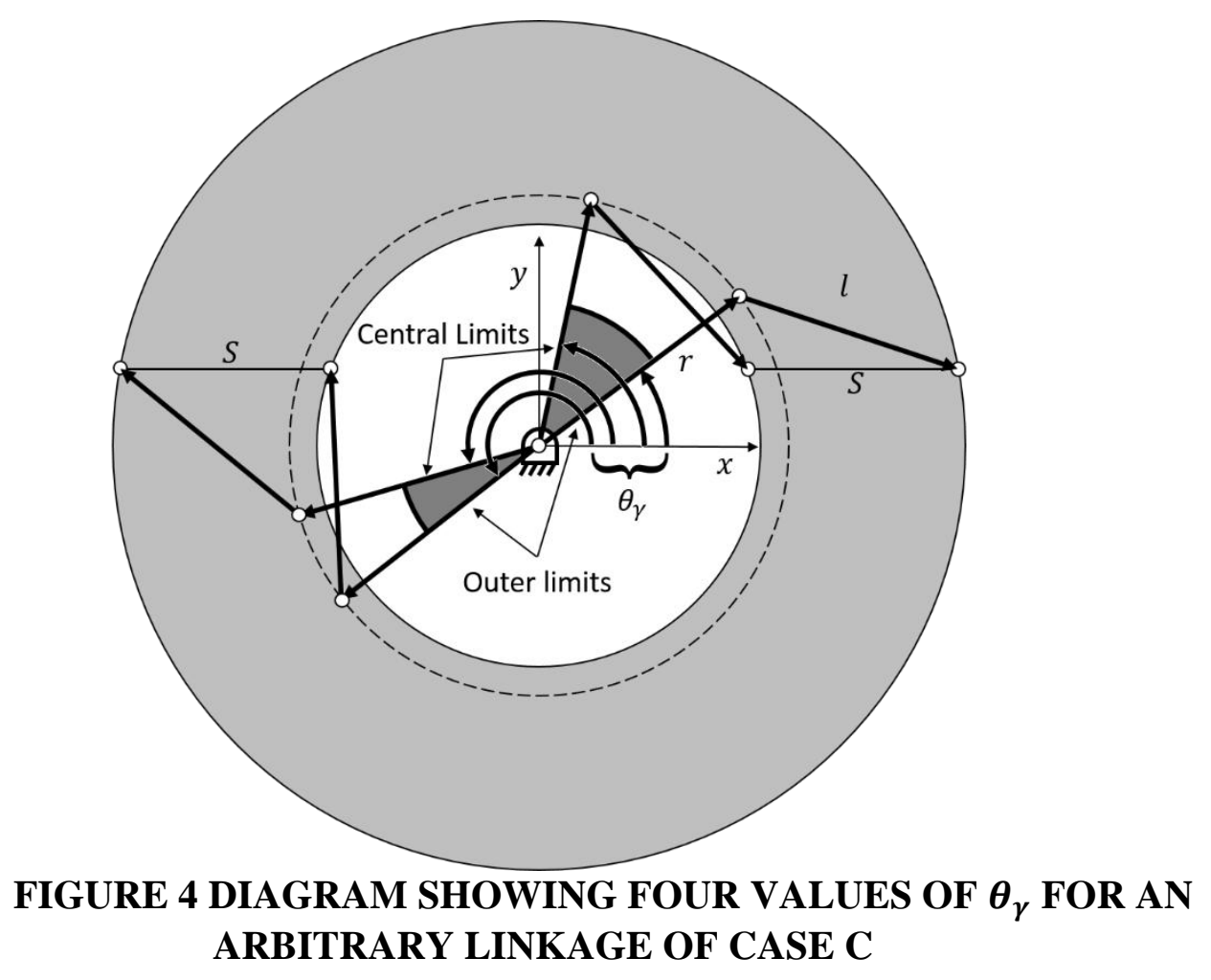

For Case $\mathrm{B}, k_{\text {min }}$ is not considered because $H \geq k_{\text {min }}$ resulting in a continuous slider segment. In this case the segment length is found by:

$$
S=2 X_{\max }
$$

There is only one unique linkage solution associated with this condition. As with case C, the above configuration of the mechanism is a mirror of the below configuration as the slider passes the $y$ axis. To find the point at which the slider crosses the $y$-axis, $X$ is set equal to zero in Eqns. (4) to (6).

\subsubsection{Determining transition through multi-valued positions}

For both Cases B and C, it is possible for the linkage to transfer between multivalued positions in which the crank changes direction as the piston continues its motion. This is because the slider dyad has no constraint on the pressure angle $\alpha$, which is allowed to pass $90^{\circ}$. As a result, the crank angle, $\theta$, as a function of $X$, can be multivalued. If a single valued result is required, these transition positions must be identified. This transition only occurs if the following conditions are met:

For the right side segment

$$
H>r \cos (\gamma)-l
$$

For the left side segment

$$
H<r \cos (\gamma)+l
$$


If either of those conditions are met the position at which the transition occurs can be found by:

$$
\begin{aligned}
& X_{0}= \pm \sqrt{r^{2}-(H \pm l)^{2}} \\
& \theta_{0}=\operatorname{atan} 2\left(H \pm l, X_{0}\right)
\end{aligned}
$$

where using the + and - in Eqns (10) and (11) corresponds to the right and left segments respectively.

Figure 5 shows the critical positons between Cases B and C where the linkage transitions through key points. For Case $\mathrm{C}$, the right and left segment solutions are identified with grey and black vectors respectively. Positions 1-4 correspond to the points of minimum transmission angle $\gamma$ or $\alpha$. Note that in this figure, $X_{0}$ occurs before the fourth position of the mechanism demonstrating that the crank angle, $\theta$, is not always increasing as the slider moves from right to left. For Case $B$, the left and right segments are part of the same mechanism. Since the slider can pass freely between the left and right sides, the interior points 2 and 3 are coincident, and are numbered this way for consistency with Case $C$. These two points however have a transmission angle $\gamma$ greater than 1 and 4 .

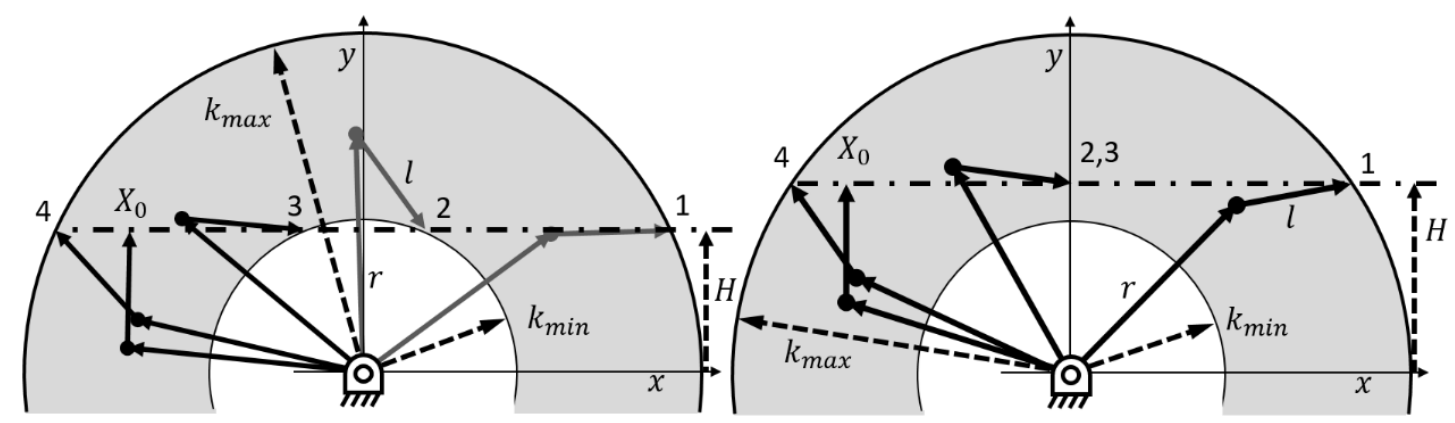

FIGURE 5 CRITICAL POSITIONS OF SLIDER DRIVEN MECHANISM FOR CASE B AND C DEPICTING THE MINIMUM TRANSMISION ANGLES OF THE CRANK DYAD AND THE TRANSITIONS TO MULTI-VALUED SOLUTIONS

\subsection{Crank Driven Mechanism}

In this section, the solution rectification of a crank driven mechanism is described in which the pressure angle $\alpha$ is constrained. A new variable, $Y$, is introduced which is the distance between the axis of slide and the joint connecting $r$ and $l$. The offset, $Y$, can be above or below the axis of slide. As seen in Figure 6, this offset is a function of $\alpha$ according to:

$$
Y=l \sin (\alpha)
$$




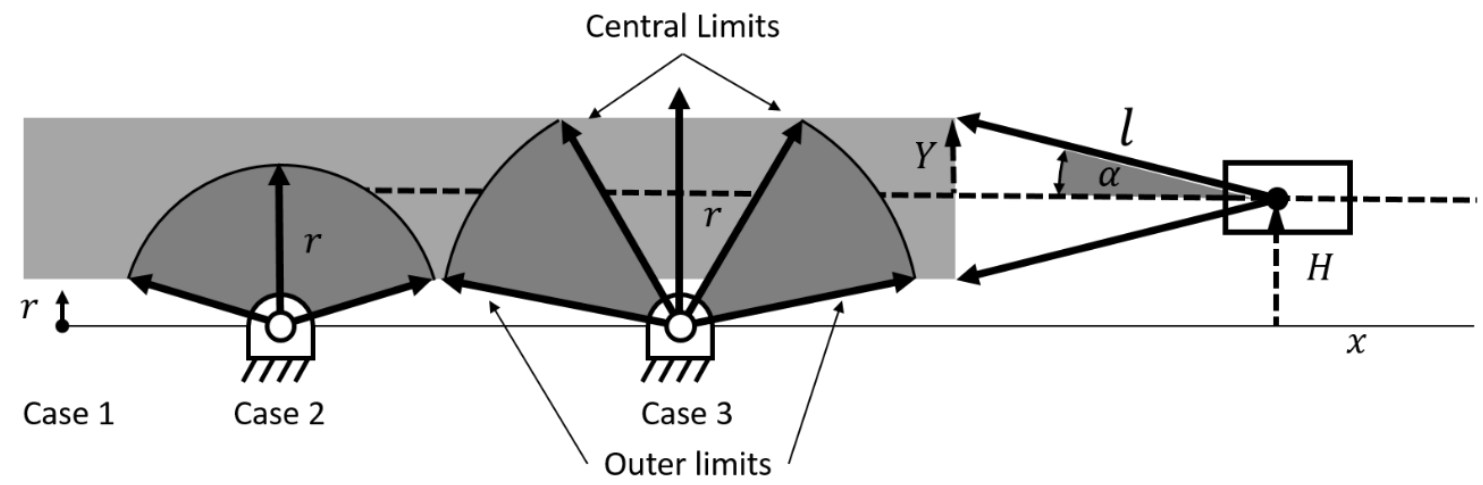

FIGURE 6 DEFINING THE VALID SLIDER POSITIONS CONSIDERING $\alpha$ CONSTRAINTS

The constraints on $\alpha$ result in four distinct solution spaces:

Case 1: If $r<(H-Y)$, the crank is too short and the constraint cannot be satisfied and no useful solution exists.

Case 2: If $(H-Y)<r \leq(H+Y)$, a continuous range of $\theta$ is possible.

Case 3: If $r>(H+Y)$, the valid range of $\theta$ is split by a region which does not satisfy the constraint resulting in two segments similar to the $\gamma$ limited Case.

Case 4: If $r<(Y-H), \alpha$ is non-constraining and need not be considered further. This is equivalent to a Grashof crank-slider mechanism with transmission angle control as the crank is allowed to make a complete revolution without breaking the constraint. This case is not shown in Figure 6.

Case 3 represents the most general solution space as it has 2 separate regions, a right and a left, defined by four angles of the crank. These right and left regions are not to be confused with the right and left segments of the slider driven mechanism. The four defining angles are categorized into central and outer limits. The solution space is depicted in Figure 6.

Figure 7 depicts the geometry used to determine the valid angles of the crank for Cases 2 and 3, which are both valid and constrained. The variable $m$ is introduced to define the right triangle $\Delta r(H \pm Y) m$. This value can then be used to determine the bounds on the angle of the crank $\theta_{\alpha}$ :

$$
\begin{gathered}
m=\sqrt{r^{2}-(H \pm Y)^{2}} \\
\theta_{\alpha}=\operatorname{atan2}((H \pm Y), \pm m)
\end{gathered}
$$


where $(H+Y)$ in Eqns (13) and (14) results in the central limits and $(H-Y)$ results in the outer limits of the valid range of $\theta_{\alpha}$. The evaluation of Eqn (14) with + or $-m$ results in the values for the right and left regions respectively.

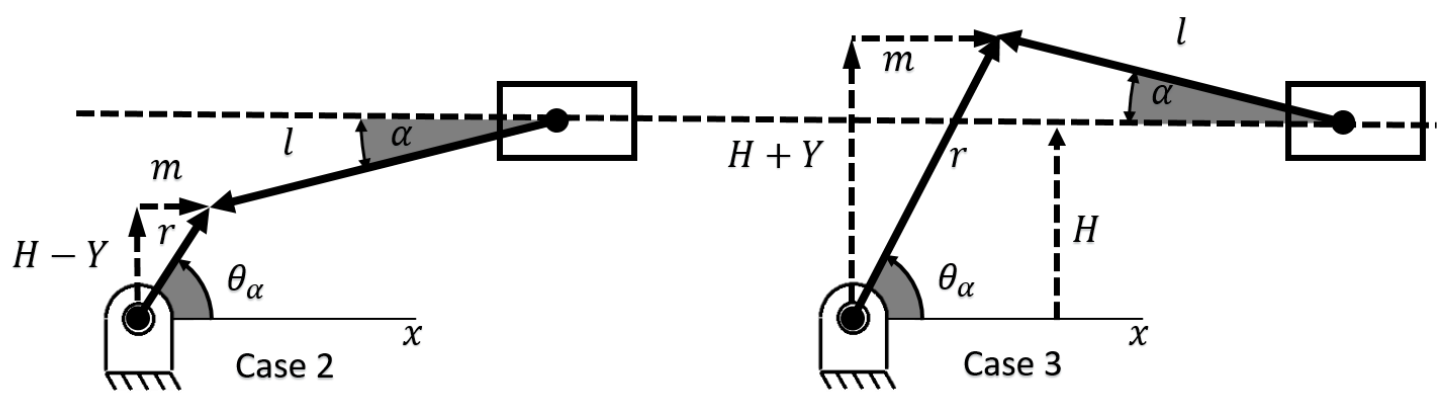

\section{FIGURE 7 DEFINING $\alpha$ LIMITED CRANK ANGLE $\theta$ FOR TWO DIFFERENT CASES OF CRANK LENGTH $r$}

There are four values of $\theta_{\alpha}$ which define the bounds of the valid regions of the crank angles $\theta$ of both the left and right region respectively. For Case 2 the central limit is not considered resulting in a continuous range of $\theta$, with the mechanism only bounded by the two outer limit values of $\theta_{\alpha}$.

\subsection{Switched Input Mechanisms}

For some mechanisms, the slider or the crank can be the driver link depending on the mode of the linkage. In these cases, both $\gamma$ and $\alpha$ should be specified and the slider driven and crank driven solution spaces must be compared. Each of the two solution spaces result in a set of four angles that bound the valid crank angles: the outer-left, central-left, central-right, and outer-right bounding angles. For Case 2, Case 4, and Case $B$, the central limits are null. These two sets of four angles must then be compared to find the limiting values. Because there is a constraint on $\alpha, \theta_{0}$ of Eqn. (11) need not be considered.

For each bounding angle comparison, there are three feasible results:

Case I: If there is no overlap, then there are no valid regions of linkage motion.

Case II: If there is overlap and all for central angles are null, the result is a continuous segment. This occurs when both Case $B$ and Case 2 true or when both Case B and Case 4 are true.

Case III: Otherwise, there will be a left side and right side range in which the mechanism can operate but cannot transfer between the two with acceptable transmission angles, forming different transmission-anglelimited pseudo-circuits of the linkage. These are not true circuits because the linkage could potentially pass from one to the other without taking the linkage apart, but this cannot occur while satisfying the transmission angle constraints. 
Figure 8 shows a sample of the overlapped solution space for an arbitrary linkage limited by both the $\gamma$ and $\alpha$ constraints. Only the right segment is shown, but a similar graphic could be made for the left segment as well. Note how the overlap is contained interior to both sets of limits.

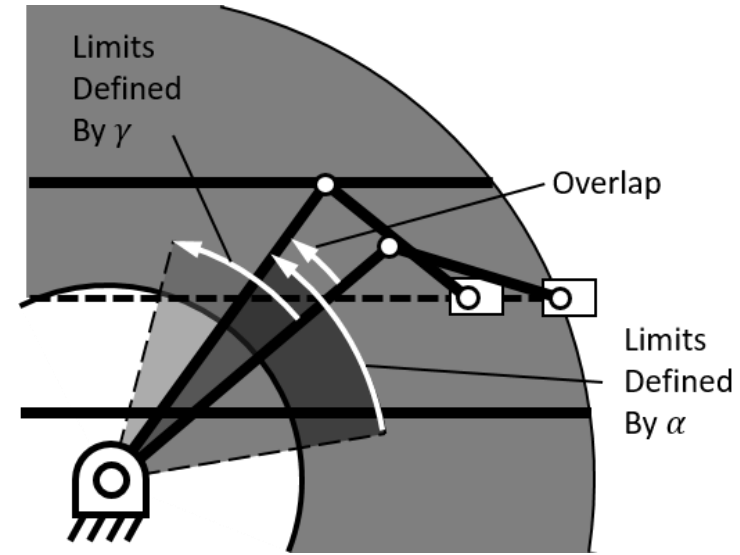

\section{FIGURE 8 OVERLAP OF THE RIGHT SIDE $\alpha$ AND $\gamma$ LIMITS FOR AN ARBITRARY LINKAGE}

\section{EXAMPLE MECHANISM}

A Watt-Il sixbar with a slider output is used as an example for clarification and to demonstrate the method. Typically, the entire mechanism is optimized simultaneously, but here the link lengths and base fourbar are provided. The slider offset height $H$ is to be optimized. As seen in Figure 9, the base fourbar of the Watt II is a Grashof crankrocker having an output rocker which travels through a $39.4228^{\circ}$ arc, and is the input of the output slider-crank mechanism. As such $R_{5}$ is considered the input crank of the slider diad. The dimensions of this linkage are given in Table 1. Note that the mechanism is normalized by the input crank length $R_{2}$. As the slider is the output link, only the pressure angle of the slider, $\alpha$, must be considered, and a maximum value of $20^{\circ}$ is imposed. However, for demonstration purposes, the minimum transmission angle, $\gamma$, is set to $20^{\circ}$ resulting in a $\gamma_{\min }$ of $20^{\circ}$, and a $\gamma_{\max }$ of $160^{\circ}$. In this example, the values of the slider offset distance, $H$, that satisfy these constraints will be determined. 


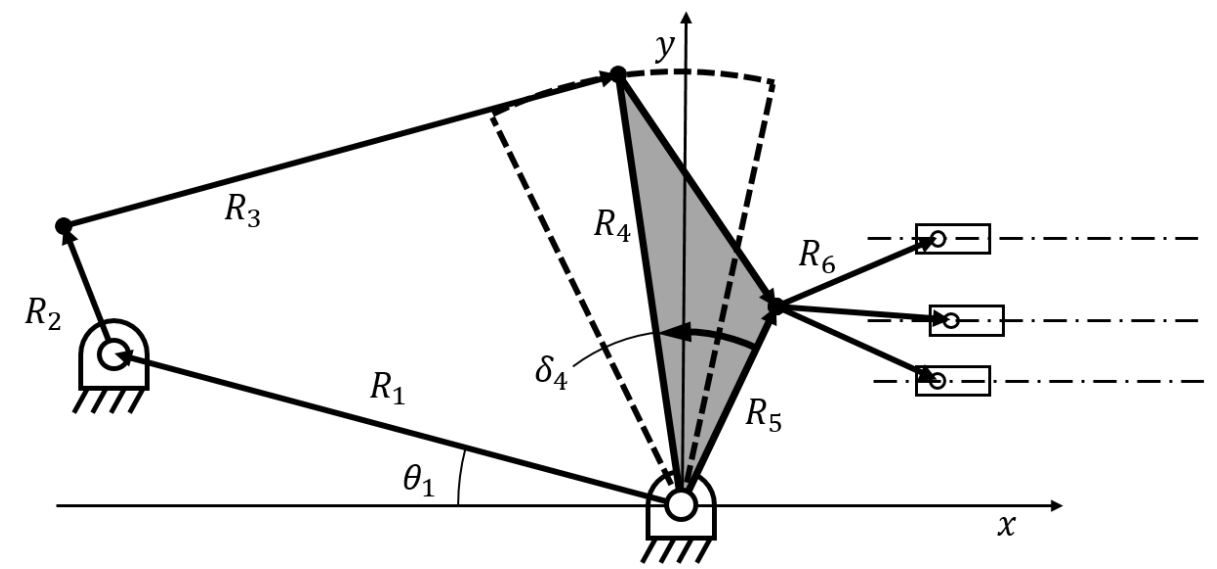

FIGURE 9 PROBLEM STATEMENT DEFINING CANDIDATE

LINKAGE

TABLE 1 PARAMETERS OF EXAMPLE LINKAGE

\begin{tabular}{|c|c|c|c|c|c|c|c|c|}
\hline Parameter & $R_{1}$ & $R_{2}$ & $R_{3}$ & $R_{4}$ & $R_{5}$ & $R_{6}$ & $\theta_{1}$ & $\delta_{4}$ \\
\hline Value & 4.38 & 1 & 4.3 & 3.13 & 1.6 & 1.3 & $14.3^{\circ}$ & $30^{\circ}$ \\
\hline
\end{tabular}

\section{Step 1: Define Slider Dyad}

The slider-crank output of the Watt II is defined by the crank length $R_{5}$, the coupler length $R_{6}$, and the swept crank angle from $\theta_{5_{0}}$ to $\theta_{5_{1}}$. Due to the rocker path generated by the crank-rocker input, the swept angle $\theta_{5}$ does not cross the $y$-axis so the right side solution for the crank driven limited space is considered.

\section{Step 2: Determine Valid Slider Axis Heights}

The limits on the slider offset due to the $\gamma$ transmission angle is found by, first, solving for $k_{\max }, k_{\min }$, and $\phi_{k}$ from Eqns (1) and (5). Next, the four values of $\psi_{k}$ are found from Eqn (6) with $\theta_{\gamma}$ set equal to either $\theta_{5_{0}}$ or $\theta_{5_{1}}$. The slider offset is described by:

$$
H=k \sin \psi_{k}
$$

where $H$ has four values associated with $\theta_{5_{0}}$ and $\theta_{5_{1}}$, using both $k_{\min }$ and $k_{\max }$. The limiting values are selected (minimum of the $k_{\max }$ value and maximum of the $k_{\min }$ value). The calculated slider offset values and resulting limits are presented in Table 2 and the resulting $\gamma$ limited solution space is shown in Figure 10. 


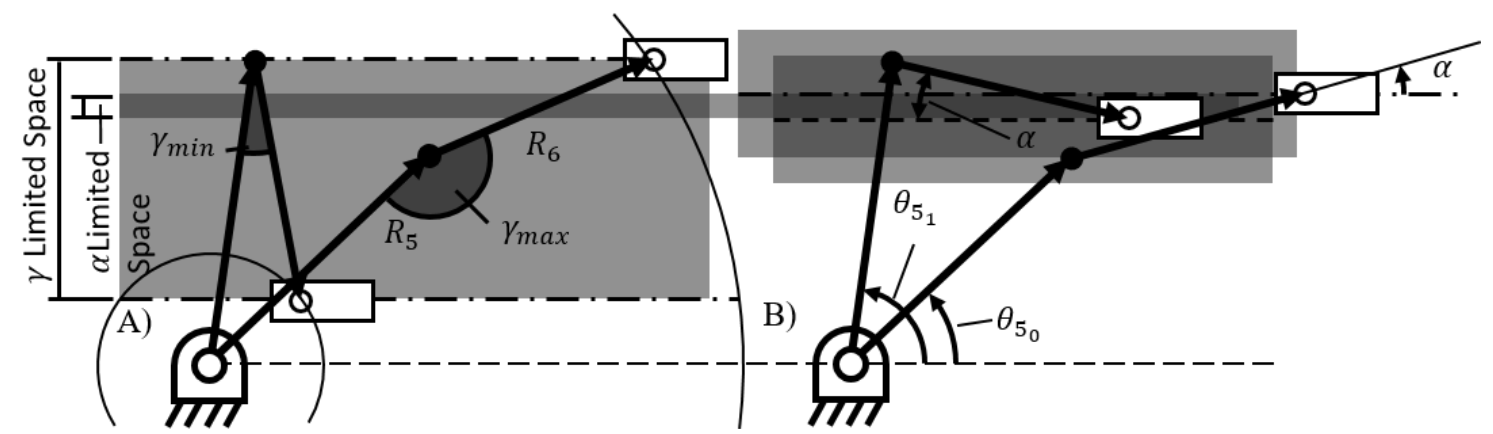

FIGURE 10 SLIDER HEIGHT SOLUTION SPACE FOR THE RIGHT SEGMENT SOLUTION SPACE

TABLE 2 CALCULATED VALUES OF $\gamma$ LIMITED VALUES OF $H$

\begin{tabular}{|c|c|c|}
\hline & $k_{\min }$ & $k_{\max }$ \\
\hline$\theta_{5_{0}}$ & $H=-.0208$ & $H=1.7824$ \\
\hline$\theta_{5_{1}}$ & $H=0.3545$ & $H=2.7943$ \\
\hline Limiting Value & $H=0.3545$ & $H=1.7824$ \\
\hline
\end{tabular}

A similar process is completed to solve for the $\alpha$ limited space. By computing $Y$ from Eqn. (12) the slider offset is described by:

$$
H=R_{5} \sin \theta_{\alpha} \pm Y
$$

where $\theta_{\alpha}$ is set equal to either $\theta_{5_{0}}$ or $\theta_{5_{1}}$ resulting in 4 values for $H$ from which the limiting values must be selected. The resulting $\alpha$ limited solution space is shown in Figure 10. The results from the $\gamma$ and $\alpha$ limited cases must be compared to determine the most limiting values of $H$, as shown in Table 3 .

TABLE 3 CALCULATED VALUES OF $\alpha$ LIMITED VALUES OF $H$

\begin{tabular}{|c|c|c|}
\hline & Min H & Max H \\
\hline$\alpha$ & 1.1532 & 1.6255 \\
\hline$\gamma$ & 0.3545 & 1.7824 \\
\hline Limiting Value & 1.1532 & 1.6255 \\
\hline
\end{tabular}

\section{Step 3: Select a Slider Axis Height}

From the range of acceptable slider offset distances, $H$ can be selected freely. If, for example, $\alpha$ were to be minimized and if $H$ is limited by $\alpha$ alone, the average of the limits of $H$ would be selected. In general, the transmission angle is smaller as the slider axis height approaches the limits. In order to maximize the transmission angle, the distance between the axis of slide and the limits is maximized. For this problem, $H$ is selected such that $\gamma$ is maximized. This corresponds to an $H$ of 1.1532 in the example with a $\gamma_{\min }$ of $48.78^{\circ}$. 


\section{Step 5: Build Solution Linkage}

The resulting sixbar mechanism is shown in Figure 11 at both ends of the slider displacement and the mid-point. This solution has the largest minimum transmission angle between links $R_{5}$ and $R_{6}$ of the right segment linkage. To achieve a specified stroke, a stretch operator can be applied to the mechanism such that the resultant $S$ is equal to the required stroke.

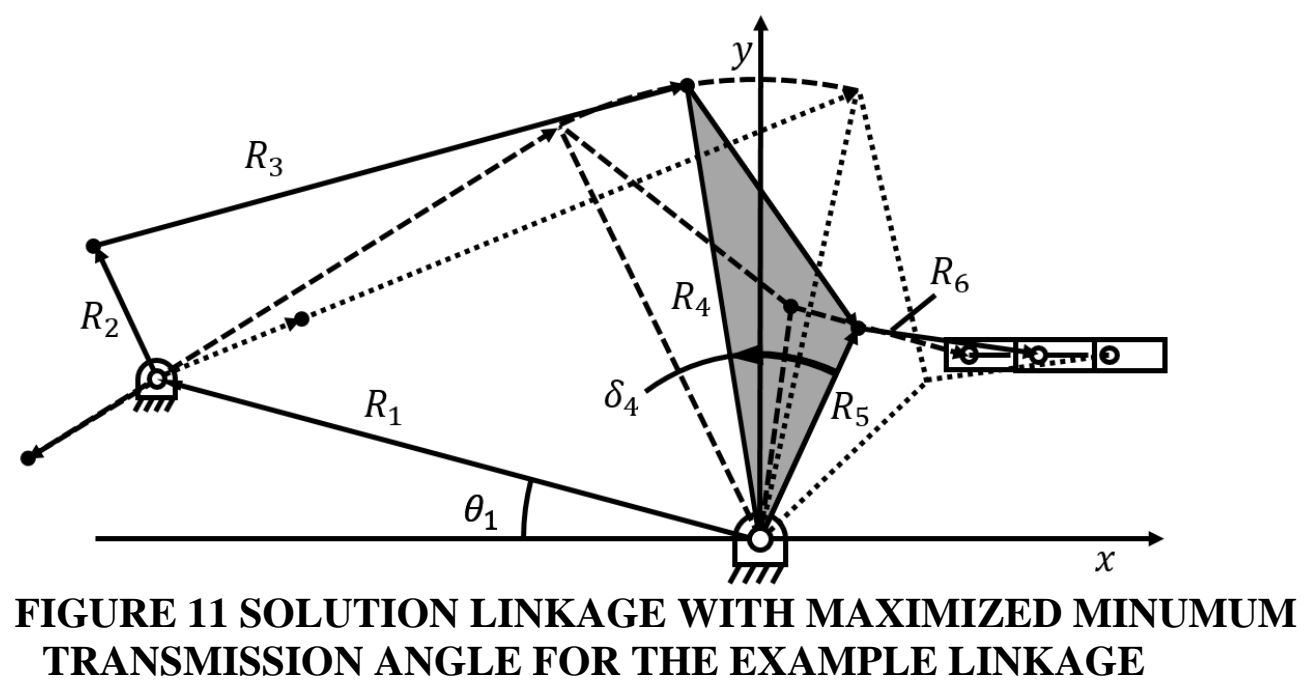

\section{CONCLUSIONS}

The range of motion as well as a method of controlling the transmission angles of a four-link slider-crank mechanism has been presented, and can be used to prevent defects. Methods for both crank driven and slider driven were presented along with a combination of the two for mechanisms which reverse the driver and driven links. By creating a lower bound on $\gamma$ or and upper bound on $\alpha$, the presented equations can be used to define the range of motion of slider-crank mechanisms. A Watt-II linkage pump mechanism was provided as an example to show how the method can be used to limit the solution space. In this case, a span of just 0.47 lengths of the input crank $\left(R_{2}\right)$ was found for the slider axis height providing a narrow band of solution space for evaluation. The example also demonstrated how different aspects of the linkage definition can be used to parameterize the linkage solution space. The general solution is not limited to this type of linkage, and can be applied to any linkage having one or more slider-crank dyads. Future work includes applying the methodology to linkages with adjustable topology, and demonstrating its use in optimization problems.

Solution rectification can be especially useful when applied to optimization problems. Typically, the inputs to an optimization are meant to be as general and unconstraining as possible. When optimizing a mechanism, the majority of evaluated linkages have defects, and are unusable as a result of this generality. While other optimization methods typically eliminate these defective solutions after they are evaluated, the equations presented here can be used to prevent their evaluation entirely. Previously, this was not possible for slider-crank mechanisms. Using only a few 
closed form equations, the evaluation of defective slider-crank mechanisms can be avoided without unnecessarily constraining the problem, thus preventing wasted computational effort, speeding up optimizations, and resulting in better solutions.

\section{ACKNOWLEDGEMENTS}

This work is supported by the National Science Foundation under grant numbers EFRI-1038294 and AIR-TT-1414053 and the Defense Advanced Research Projects Agency Under Contract \# W31P4Q-11-C-0060.

\section{REFERENCES}

[1] S.S. Balli, S. Chand, Defects in link mechanisms and solution rectification, Mech. Mach. Theory. 37 (1999) 851-876.

[2] S.S. Balli, S. Chand, Transmission angle in mechanisms (Triangle in mech), Mech. Mach. Theory. 37 (2000) 175-195.

[3] J.E. Holte, T.R. Chase, A force transmission index for planar linkage mechanisms, in: Proc. ASME Mech. Conf., 1992: pp. 377-386.

[4] C.-C. Lin, W.-T. Chang, The force transmissivity index of planar linkage mechanisms, Mech. Mach. Theory. 37 (2000) 1465-1485.

[5] W.-Y. Lin, K.-M. Hsiao, Optimum synthesis of a 10-link gripping mechanism using new grasping indices, J. Chin. Inst. Eng. (2016) 1-7. doi:10.1080/02533839.2016.1187086.

[6] B. He, P. Zhang, L. Liu, Simultaneous functional synthesis of mechanisms with mechanical efficiency and cost, Int. J. Adv. Manuf. Technol. 75 (2012) 659-665.

[7] T.A. Sullivan, W.F. Northrop, K. McCabe, Integrated Mechanical and Thermodynamic Optimization of an Engine Linkage Using a Multi-Objective Genetic Algorithm, J. Mech. Des. 137 (2012) 024501-1.

[8] T. Sullivan, Multi-domain multi-objective optimization of mechanisms: a general method with two case studies, UNIVERSITY OF MINNESOTA, 2011.

[9] J.A. Cabrera, A. Simon, M. Prado, Optimal synthesis of mechanisms with genetic algorithms, Mech. Mach. Theory. 37 (2000) 1165-1177.

[10] W.-Y. Lin, A GA-DE hybrid evolutionary algorithm for path synthesis of four-bar linkage, Mech. Mach. Theory. 45 (2008) 1096-1107.

[11] H. Lipson, Evolutionary synthesis of kinematic mechanisms, Artif. Intell. Eng. Des. Anal. Manuf. 22 (2006) 195-205.

[12] W.Y. Lin, C.L. Shen, K.M. Hsiao, A Case Study of the Five-Point Double-Toggle Mould Clamping Mechanism, Proc. Inst. Mech. Eng. Part C: J. Mech. Eng. Sci. 220 (2006). doi:10.1243/09544062JMES216.

[13] W.Y. Lin, S.S. Wang, Dimensional synthesis of a five-point double-toggle mould clamping mechanism using a genetic algorithm--differential evolution hybrid algorithm, Proc. Inst. Mech. Eng. Part C: J. Mech. Eng. Sci. 224 (2010). doi:10.1243/09544062JMES1951.

[14] S. Ebrahimi, P. Payvandy, Efficient constrained synthesis of path generating fourbar mechanisms based on the heuristic optimization algorithms, Mech. Mach. 
Theory. 85 (2015). doi:10.1016/j.mechmachtheory.2014.11.021.

[15] V.H. Alt, The Transmission Angle and its Importance for the Design of Periodic Mechanisms, Werstattstechnik. 26 (1930) 61-64.

[16] F. Freudenstein, E.J.F. Primrose, The classical transmission angle problem, in: Proc. Conf. Mech., 1971: pp. 105-110.

[17] E. Söylemez, Classical transmission-angle problem for slider-crank mechanisms, Mech. Mach. Theory. 37 (2000) 419-425.

[18] A.F. Vadasz, Design charts for slider crank mechanism with prescribed minimum transmission angle and time ratio, in: Proc. 6th Annu. Appl. Mech. Conf., 1976: pp. XXXI-1-XXXI. 